\title{
MICROSCALE ELECTROLYTIC PROBES, WITH INTEGRATED TEMPERATURE SENSORS, FOR NANO- TO MICRO-LITER FLUID VOLUMES
}

\author{
Dongming He \\ Assembly Technology Development, Intel Corp. \\ Chandler, AZ 85224
}

\author{
Mark A. Shannon ${ }^{1}$ \\ University of Illinois at Urbana-Champaign \\ Urbana, IL 61801
}

\begin{abstract}
Electrolytic conductivity measurements of fluids currently require sample volumes greater than a milliliter. Many applications would benefit from accurate measurements for nano- to microliter sample volumes. However, polarization and non-linear electrode impedance, along with stray impedance's and temperature effects, strongly affect measurements of the solution resistance for microliter and smaller sample volumes. MEMS-based $\mathrm{Si}$ electrolytic conductivity probes, down to $50 \mu \mathrm{m}$ wide, with integrated temperature sensors have been designed and fabricated to mitigates these issues. The accuracy of normal scale probes is achieved with these sensors over 3 orders of magnitude in solution concentration.
\end{abstract}

\section{INTRODUCTION}

Electrolytic sensors can have extensive applications in chemical and biological testing, such as pharmacology, blood and urea analysis, and tissue perfusion measurements. However, conventional conductivity probes are too bulky and blunt for insertion into tissue, and most importantly require relatively large sample volumes to make accurate measurements. In particular, the cell constant, $K_{c}$, of the conductivity probe varies with the geometry of the probe and the volume being sampled. Thus, as the sample size is reduced for a specific probe size, the measured solution resistance, $R_{s}$, does not linearly vary with the conductivity below a critical dimension, and substantial errors can result. Often, the critical dimension to maintain a constant $K_{c}$ is approximately one order of magnitude larger than the effective electrode spacing, $L_{\text {eff, }}$ of the probe. Therefore, the volume occupied by the probe needs to be about 3 orders of magnitude smaller than the sample volume. Currently, for 96 well arrays, probe tips are needed that can accurately sample microliter sample volumes. The much larger $300+$ arrays coming on-line need 10 nanoliter sampling! Electrolytic probes with such small dimensions are currently unavailable, and simply reducing their size with designs that have been reported in the literature or are commercially available leads to low sensitivity and large errors in the measurements.

Electrolytic probes used for tissue insertion present another set of challenges. Not only is the volume of solution inherently confined to around the probe tip, but the shape and structure must also allow insertion and sustain relatively large stresses and strains. In addition, electrolytic conductivity is a strong function of temperature, $1-3 \%$ per ${ }^{\circ} \mathrm{C}$ for most electrolyte solutions. Since skin temperature can vary on the order of $10^{\circ} \mathrm{C}$ from the core body temperature and is strongly dependent on depth, the temperature at the point of measurement needs to be known. Ideally, high-aspect ratio electrolytic probes of $\sim 100 \mu \mathrm{m}$ width are needed, with local temperature compensation. Current methods use needles for extraction and often need several milliliters of fluid. Microscale silicon probes on the order of $100 \mu \mathrm{m}$ in cross-section are easily insertable, are strong, and can sustain relatively high strains. Microscale probes are also needed that can physically measure the local temperature and concentration in a fluid boundary layer within $100 \mathrm{~mm}$ of the surface with minimal flow disturbance Simply put, new types of microscale electrolytic probes are needed that meet all the above criteria for a wide variety of applications.

(a)

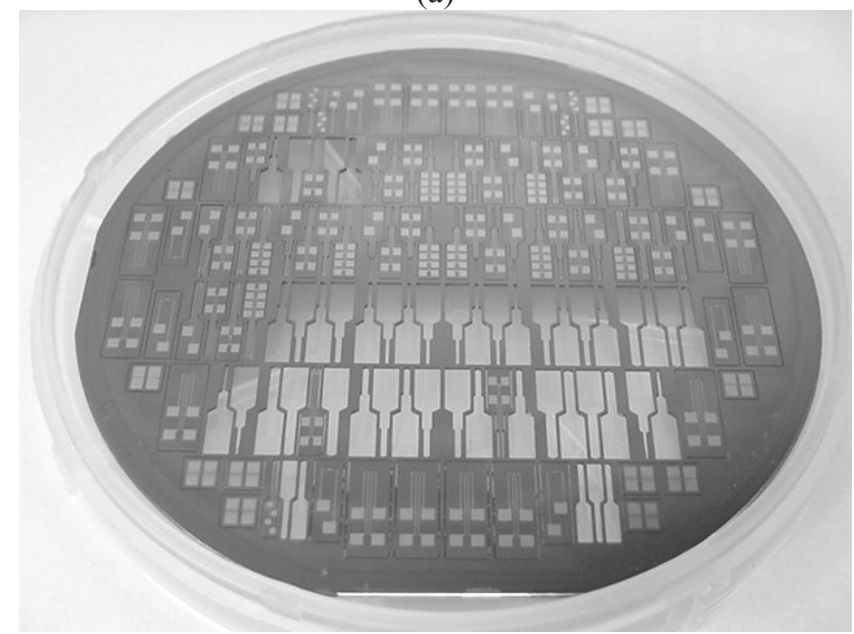

(b)

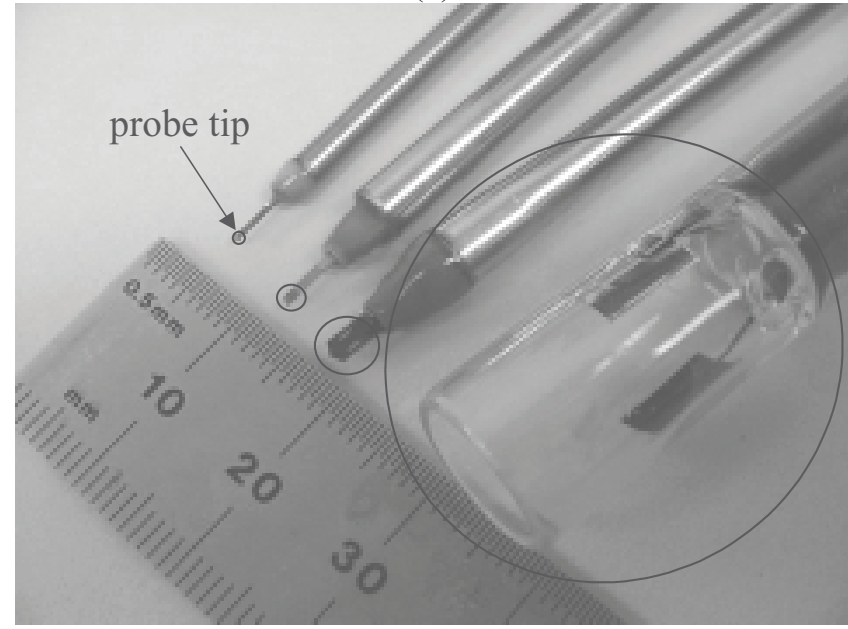

Figure 1. (a) Photo of a wafer fabricated Si probe tips, with some probes removed from the wafer. Note that the large end accommodates large bond pads for hand soldered leads. Solderbump pads make the overall size the same as the probe. (b) $A$ photo showing sensors of 500, 1000, and $2000 \mu \mathrm{m}$ tip width after wire bonding and packaged with stainless tubes, and a commercial 2-electrode conductivity sensor probe.

\footnotetext{
${ }^{1}$ Submitting author. Address: 1206 West Green Street, Urbana, IL 61801, USA. Email: mas1@uiuc.edu
} 
Silicon conductivity sensors have been reported with smooth 2 and 4-electrode probe tips for relatively $(\sim 1 \mathrm{~mL})$ small volume analysis [1-5]. However, smooth $\mathrm{Au}$ or $\mathrm{Pt}$ microelectrodes have high polarization impedance, making good accuracy difficult if their size is decreased further. In addition, stray capacitance, lead resistance, and high-frequency effects severely limit sensitivity and accuracy as tip sizes drop below $\sim 1 \mathrm{~mm}$. To solve these problems we developed several new design and processing parameters, and fabricated mass-producible, $1 \mathrm{kHz}$, silicon microelectrolytic sensors with temperature compensation, shown in Fig. 1. These are more than an order of magnitude smaller than other conductivity probes, accurately measuring $\mu \mathrm{L}$ to $\mathrm{nL}$ samples. Moreover, the linear conductivity response of the 4-electrode Pt-black sensors ranges over three orders of magnitude, providing better performance than large commercial 2-electrode probes. Therefore, these MEMS-based electrolytic probes offer a viable solution to the vexing problem of nanoliter sample problems, as well as creating insertable probes for tissue and boundary layer flows.

\section{THEORY}

Electrolytic conductivity sensors essentially work by measuring the resistance to current flow across electrodes in an ionic solution. Both conduction current and displacement currents can occur at the electrode/solution interface. To avoid chemical reactions associated with conduction current, nearly ideal polarized electrodes [6], such as $\mathrm{Au}$ and $\mathrm{Pt}$, are often utilized for conductivity measurements with $\mathrm{AC}$ excitations to generate a displacement current. However, precise conductivity measurements at the microscale can be strongly affected by polarization impedance, which is a function of the driving frequency, electrolytic concentration and electrical potential. Nonlinear impedance effects, such as ion diffusion, chemical reactions, adsorption and desorption of ions at the electrode/solution interface, become more important at high potential and current density, creating a non-constant interfacial impedance during each $\mathrm{AC}$ voltage swing. Increasing electrode area and reducing the applied potential help mitigate these effects, but often at the expense of increasing overall device size and increasing operational frequency, both of which acerbate the effect of stray capacitance and non-linear impedance at the microscale.

Figure 2 shows the layout of the sensor electrodes that use four planar electrodes, along with the major dimensions and current paths through the ionic solution. Both the layout of the sensor and the current path, which is affected by the overall geometry and volume of the sample volume, determine the resistance through the solution, which gives a direct measure of the solution conductivity and hence it's ionic concentration. To determine the sensor resistance, $R_{s}$, a simplified equivalent circuit of the electrodes is shown in Fig. 3. Each electrode generates a double layer capacitance with the ionic solution, which is a function of the surface potential and ionic concentration, $c$.

The measured solution resistance, $R_{S}$, is often set equal to $K_{C} / \sigma$, where $K_{C}$ is the cell constant that depends on geometry and $\sigma$ is the solution conductivity to be measured. The main issue for microscale sensors is that $K_{C}$ can grow rapidly as the size of the sensor is reduced, due to a relative increase in electrode impedance, thereby reducing the accuracy of the measured $R_{s}$. Therefore, the electrode layout needs to be designed to reduce $K_{C}$, while considering the overall geometry of the probe and the paths that the current take within the solution. (a)

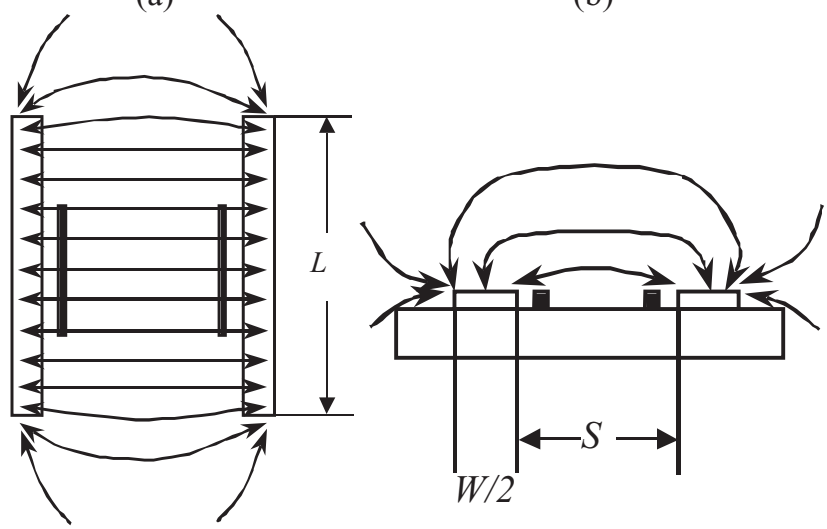

Figure 2. The top (a) and side (b) view of a planar 4-electrode configuration, showing critical dimensions and current path through a surrounding fluid.

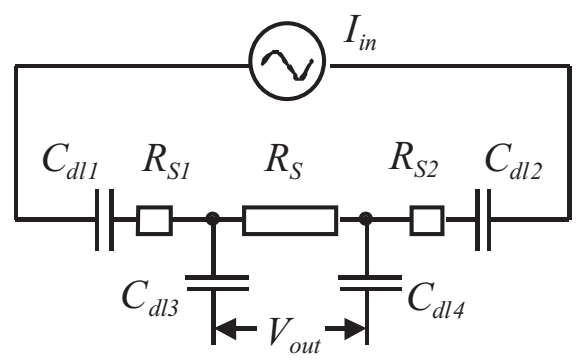

Figure 3. A circuit representation of the electrode impedences for the 4-electrode sensor in Fig. 2. Note that stray capacitances and resistances of the leads, as well as $Z_{f}$, are not included.

The current flow in Fig. 2 is three-dimensional and requires numerical modeling. However, when $L \gg S$ and $W$ then $K_{C}=$ $\left(R_{S I}+R_{S}+R_{S 3}\right) \sigma$ can be expressed as

$$
\begin{gathered}
K_{C}=\frac{2 \varphi(a)}{L \varphi\left[\left(1-a^{2}\right)^{\frac{1}{2}}\right]} \\
\varphi(a)=\int_{0}^{1} \frac{d t}{\left[\left(1-t^{2}\right)\left(1-a^{2} t^{2}\right)\right]^{\frac{1}{2}}}
\end{gathered}
$$

where $\varphi$ is the potential, and $a=S /(S+W)$. The results for calculated $K_{C}$ using Eqn. (1) are 17 to $34 \%$ higher than the measured due to neglecting 3-D current flow effects. The 2-D design using Eqn. (1) provides a upper bound on $K_{C} \cdot K_{C}$. ranged from a low of $8.7\left\{\mathrm{~cm}^{-1}\right\}$ for $2000 \mu \mathrm{m}$ wide tip probes to approximately $44\left\{\mathrm{~cm}^{-1}\right\}$ for $50 \mu \mathrm{m}$ wide tip probes. It should also be noted that polarization impedance scales inversely proportional to the effective electrode surface area $(L W / 2$ for smooth metal electrodes), and the measured solution resistance scales inversely proportional to $L$ if parameter $a$ is fixed. This implies that polarization impedance is more important for conductivity measurement with microelectrodes than normal scale devices. Therefore, polarization impedance also needs to be considered while limiting $K_{C}$ in designing smaller electrodes. Leakage currents capacitively coupled through unexposed leads at the sensor tip and the leads going to the amplifier also create stray capacitances and stray resistances. Robust packaging for the sensor tip and fabricating well-insulated leads that are as short as possible from the amplifier are also important to reduce the stray impedances. 


\section{EXPERIMENTAL DETAILS}

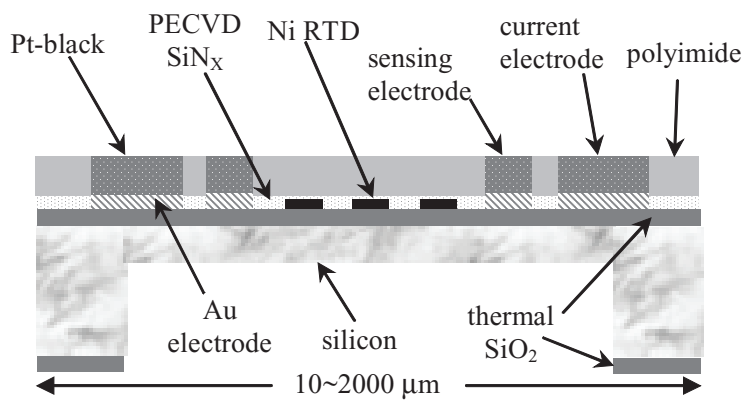

Figure 4. Cross sectional diagram of 4-electrode sensor probe tip (not to scale). Note that the $\mathrm{SiN}_{x}$ and polyimide passivation layers are needed to achieve the desired performance. In addition, the polyimide layer acts as Ptblack electroplating molds, DRIE mask, and plastic packaging of the tip.

Figure 4 shows the cross section of a probe tip fabricated by double-sided deep reactive ion etching (DRIE). Each of the layers is deposited on the wafer before etching. Several passivation and isolation layers are added to reduce stray capacitance to the substrate and to the solution. Two layers of PECVD $\mathrm{SiN}_{\mathrm{x}}$ are grown on the metal thin-films for passivation of the RTD and leads. A layer of polyimide is applied to improve isolation and to serve as packaging for the probe tip, as well as to provide the mask for double-sided DRIE etching of the wafer. Gold is evaporated and patterned for leads, microelectrodes, and bonding pads to reduce stray resistance. The electrodes are placed over a thinned silicon section to reduce thermal mass and to improve the temperature measurements. Figure 5 shows the top and side view of typical probes made with this method. Probes can be packaged in stainless tubes and wires are bonded to pads and connector pins to facilitate test and handling, as in Fig. 1.

To reduce the effect of polarization impedance, the electrodes are electroplated with Pt-black to increase effective surface area. The effective surface area of an electrode has been reported to increase from hundreds to even one thousand times after Pt-black electroplating [7]. In addition, 2 current carrying and 2 sensing electrodes were fabricated to use 4-wire measurements with $>30 \mathrm{M} \Omega$ impedance across the sensing electrodes in order to minimize the effect of polarization impedance on the measured potential.

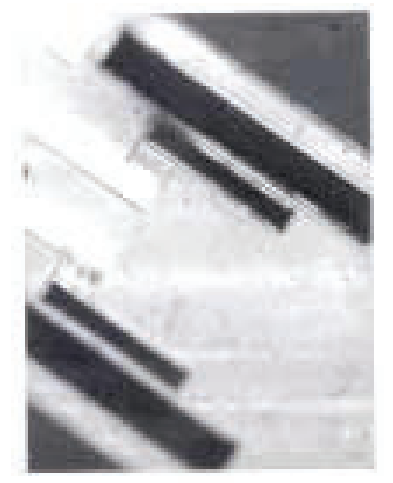

Figure 6. A $500 \mu \mathrm{m}$ sensor tip after Pt-black electroplating. Pt is plated within polyimide wells to define the plating mold and to provide additional spacing to reduce solution stray capacitance to the leads. Note that the leads are $A u$ plated to further reduce stray resistances. (a)

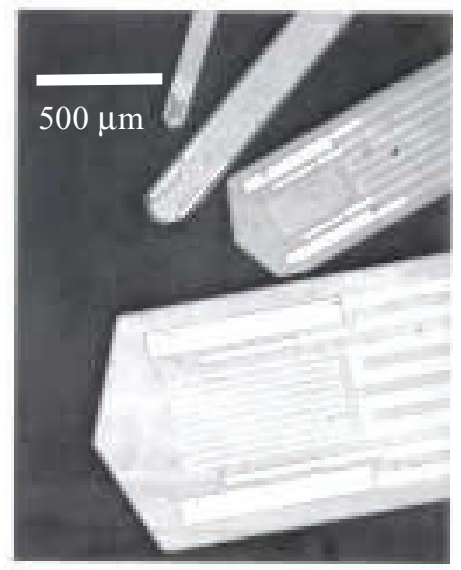

Figure 5. (a) Sensor probes of 100, 200, 500 and $1000 \mu \mathrm{m}$ tip width before Pt-black plating. (b) Side view of sensor tip is etched back to about $75 \mu \mathrm{m}$.

\section{RESULTS and DISCUSSION}

To provide a means to measure the local temperature of the solution, which is important to achieve accurate conductivity measurements, an integrated thin-film resistive temperature device (RTD) is fabricated directly between the electrodes, as seen in Fig. 5. This location corresponds to the local average temperature for conductivity measurements, even if there is a temperature gradient between the electrodes. Thin-film nickel is deposited and patterned for the RTD due to its higher temperature coefficient of resistance (TCR) than Pt. In the temperature range we are interested in (approximately from -10 to $100^{\circ} \mathrm{C}$ ), pure bulk Ni has a TCR of $6900 \mathrm{ppm}$ per ${ }^{\circ} \mathrm{C}$, compared with $3900 \mathrm{ppm}$ per ${ }^{\circ} \mathrm{C}$ for pure bulk Pt. The RTD measurements achieved better than $1 \%$ accuracy from 0 to $100^{\circ} \mathrm{C}$ after calibration.

To note the effect of polarization impedance on microscale electrolytic sensors, conductivity measurements for a $200 \mu \mathrm{m}$ wide sensor probe with 2 and 4-electrodes (with and without Pt-black) are shown in Fig. 7. Note that in Fig. 7(a) the smooth 2-electrode sensors have the largest non-linearity at highest concentrations, which can not be readily observed directly from the resistivity data in Fig. 7(b), but which nevertheless increases the uncertainty in the concentration measurement. By using Pt-black, this uncertainty is decreased to less than $4 \%$ with respect to the $95 \%$ confidence limit. In addition to reducing uncertainty, Pt-black electrodes also greatly reduces long term drift. Smooth polarized electrodes are very sensitive to fouling and ion absorption, which causes drift in the resistance measurement over time. The increase in area proportionally increases the time over which drift is noticed. However, by using sensing electrodes in a 4-wire configuration, the effect of fouling, etc. on drift is greatly diminished, since the change in impedance at the electrode interface is negligibly small compared to the input impedance of the instrumental amplifier.

As the sensor is reduced below a $100 \mu \mathrm{m}$ in size, the effect that operation frequency has on the impedance increases in importance. Figure 8 shows the square of the total impedance, $Z_{\text {tot }}{ }^{2}$ vs. $1 / \omega^{2}$, for different solution concentrations. Note that at lower frequency (higher $\left.1 / \omega^{2}\right)$ the capacitance is approximately constant, but begins to increase as frequency goes much above $10^{4} \mathrm{~Hz}$, and thus uncertainty in $R_{S}$ rapidly grows particularly at higher ionic concentrations. As the inset in Fig. 8 shows, this effect leads to non-linear response in resistivity, taking on a quadratic, rather than linear, form. Therefore, for the smallest sizes, higher order calibrations are required to achieve the highest accuracy. 


\section{CONCLUSIONS}

Microscale electrolytic probes with integrated temperature sensors were designed and fabricated to measure local conductivity and temperature of ionic solutions [8]. The Ni thin-film RTD achieved better than $1 \%$ accuracy from 0 to $100^{\circ} \mathrm{C}$ with a parabolic correlation. Two-electrode conductivity measurements with Pt-black electroplated microelectrodes achieved better than 5\% linearity for solutions with conductivity varying from 2 to $180 \mathrm{mS} / \mathrm{cm}$. However, bare 2-electrode measurements suffer from large polarization impedance and drift over time. The polarization impedance decreases hundreds-fold with Pt-black electroplating. Bare 4-electrode measurements also reduce polarization impedance and achieved better than $4 \%$ accuracy for the same measurement range for probes above $100 \mu \mathrm{m}$ tip size. Combining 4 electrode and Pt-black electrodes allows sensors to be fabricated below $100 \mu \mathrm{m}$ with better than $5 \%$ accuracy for a quadratic fit. The $50 \mu \mathrm{m}$ wide sensor can sense approximately $100 \mathrm{~nL}$ sample volume. The lower limit in size appears only to be fabrication limited, to about a $10 \mu \mathrm{m}$ wide sensor, for a $4 \mathrm{~nL}$ sample volume.

\section{ACKNOWLEGEMENTS}

This work was supported by DARPA DSO under DABT63-98-C-0053.

\section{REFERENCES}

1. W. Olthuis, A. Volanschi, J.G. Bomer, and P. Bergveld, "A new probe for measuring electrolytic conductance", Sensors \& Actuators B 13-14, 230-233 (1993).

2. N. Kordas, Y. Manoli, W. Mokwa, \& M. Rospert, "A CMOS-compatible monolithic conductivity sensor with integrated electrode", Sensors \& Actuators A 43, 31 (1994). 3. A. Volanschi, W. Olthuis, and P. Bergveld, "Design of a miniature electrolyte conductivity probe using ISFET's in a four point configuration", Sensors \& Actuators B 18-19, 404-407 (1994).

4. W. Olthuis, W. Streekstra, and P. Bergveld, "Theoretical and experimental determination of cell constants of planar-interdigitataed electrolyte conductivity sensors", Sensors \& Actuators B 24-25, 252-256 (1995).

5. N.F. Sheppard Jr. and D.J. Mears, "Model of an immobilized enzyme conductimetric urea biosensor", Biosensors \& Bioelectronics 11, 967-979 (1996).

6. J.O. Bockris and A.K.N. Reddy, Modern Electrochemistry, Plenum Press (1970).

7. R.A. Robinson and R.H. Stokes, Electrolyte Solutions, Butterworths Scientific Publications (1955).

8. D. He, "Theory, fabrication and characterization of micromachined electrolytic solution conductivity sensors," Ph.D. Dissertation, UIUC, (2001).

Figure 8. Impedance for a Pt-black 4-electrode $50 \mu \mathrm{m}$ sensor tip as a function of frequency, showing the effect of electrode capacitance on the total impedance. Inset shows $R_{S} v s$. the solution resistance. (a)

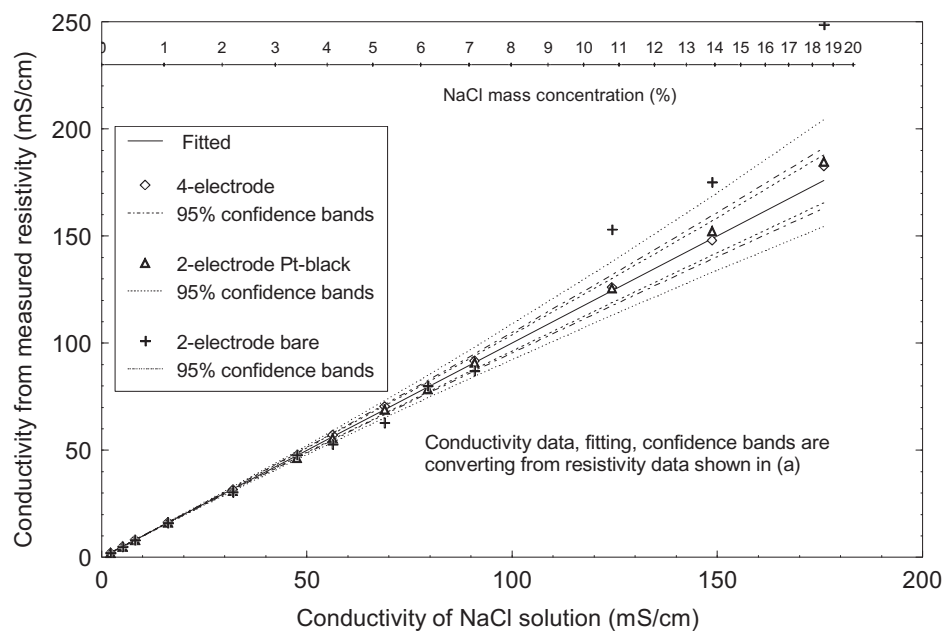

(b)

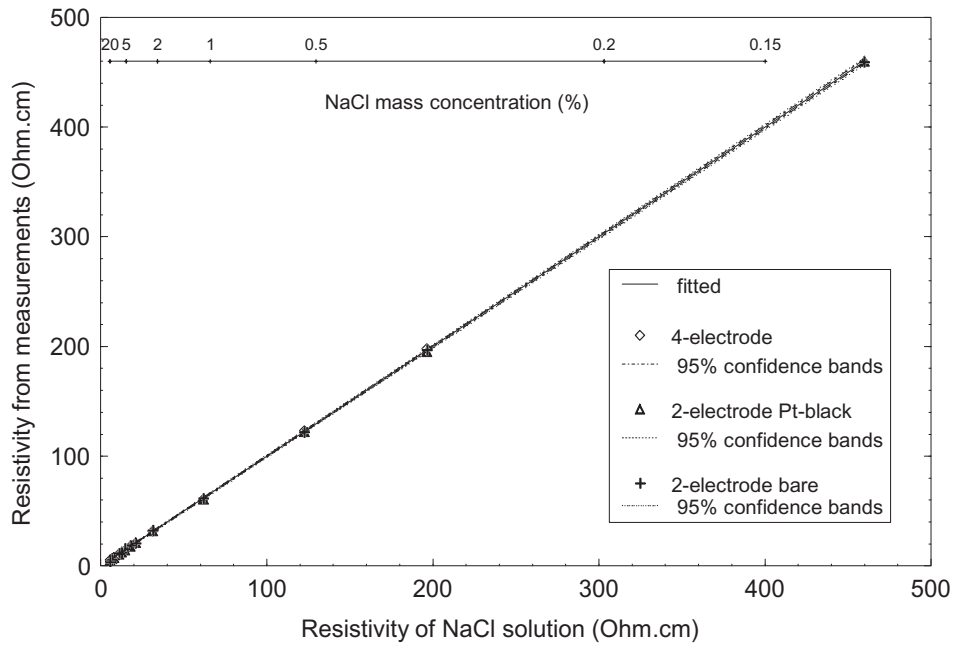

Figure 7. Experimental results normalized with linear fitting with respect to $\mathrm{NaCl}$ solution (a) resistivity and (b) conductivity for three sensor probes: 4-electrode probe without Pt-black, 2-electrode probe with Ptblack, and 2-electrode probe without Pt-black. (data all compensated to $\left.18{ }^{\circ} \mathrm{C}\right) . \mathrm{NaCl}$ molar mass \% concentration is also given, so that linearity can be discerned over the entire range with both plots. Note that both Ptblack and 4-wire measurements reduce the non-linearity, extending the linear measurement range.

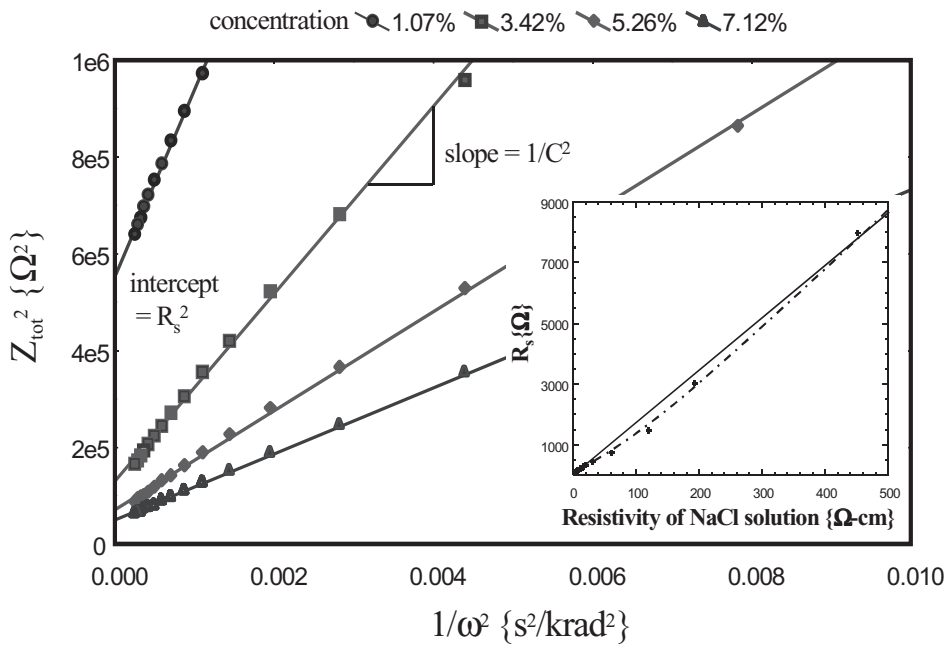

